\title{
Recruitment and Baseline Characteristics of Participants in the "Sanadak" Trial: A Self-Help App for Syrian Refugees with Post-traumatic Stress
}

\author{
Susanne Röhr ${ }^{1,2, *,+}+$ D , Franziska U. Jung ${ }^{1, \dagger}{ }^{+}$Anna Renner ${ }^{3}$, Anna Plexnies ${ }^{3}$, Rahel Hoffmann ${ }^{3}$, \\ Judith Dams ${ }^{4}$, Thomas Grochtdreis ${ }^{4}\left(\mathbb{D}\right.$, Hans-Helmut König ${ }^{4}\left(\mathbb{D}\right.$, Anette Kersting ${ }^{3}$ \\ and Steffi G. Riedel-Heller ${ }^{1}$ \\ 1 Institute of Social Medicine, Occupational Health and Public Health (ISAP), Medical Faculty, \\ University of Leipzig, 04103 Leipzig, Germany; franziska.jung@medizin.uni-leipzig.de (F.U.J.); \\ steffi.riedel-heller@medizin.uni-leipzig.de (S.G.R.-H.) \\ 2 Global Brain Health Institute (GBHI), Trinity College Dublin, D02 PN40 Dublin, Ireland \\ 3 Department of Psychosomatic Medicine and Psychotherapy, University Medical Center Leipzig, \\ 04103 Leipzig, Germany; anna.renner@medizin.uni-leipzig.de (A.R.); pt.plexnies@gmail.com (A.P.); \\ rahelhoffmann@posteo.de (R.H.); anette.kersting@medizin.uni-leipzig.de (A.K.) \\ 4 Department of Health Economics and Health Services Research, Hamburg Center for Health Economics, \\ University Medical Center Hamburg-Eppendorf, 20246 Hamburg, Germany; j.dams@uke.de (J.D.); \\ t.grochtdreis@uke.de (T.G.); h.koenig@uke.de (H-H.K.) \\ * Correspondence: susanne.roehr@medizin.uni-leipzig.de; Tel.: +49-341-9724568; Fax: +49-341-9715409 \\ + These two authors contributed equally.
}

Received: 9 September 2020; Accepted: 14 October 2020; Published: 18 October 2020

\begin{abstract}
Many Syrian refugees residing in Germany have been exposed to traumatizing events, while treatment options are scarce. Therefore, the self-help app "Sanadak" was developed to target post-traumatic stress in Syrian refugees. We aimed to inspect the recruitment and baseline characteristics of the participants in the trial, which is conducted to evaluate the app. Analyses were based on the recruitment sample $(n=170)$ and the trial sample $(n=133)$. Data were collected during structured face-to-face interviews in the Arabic language. Targeted outcomes included post-traumatic stress (primary; Post-traumatic Diagnostic Scale for DSM-5/PDS-5) and depressive symptoms, anxiety, resilience, among others (secondary). Recruited individuals were $M=32.8$ ( $\mathrm{SD}=11.2$, range $=18-65$ ) years old; $38.8 \%$ were women. The average PDS-5 score was $23.6(\mathrm{SD}=13.2)$ regarding trauma exposure, which was most frequently related to experiencing military- or combat-related events (32.9\%). Moreover, $46.5 \%$ had major depression and 51.8\% showed low resilience. Anxiety was present in $40.6 \%$ of the trial participants. Psychological distress was high in Syrian refugees residing in Germany, enrolled in a trial targeting post-traumatic stress. This underlines the need for intervention. Our results provide important figures on the mental health of a not well-studied population group in Germany.
\end{abstract}

Keywords: Syrian refugees; posttraumatic stress; eHealth; mHealth; app; smartphone; trial; randomized-controlled trial; intervention; baseline; mental health

\section{Introduction}

About 790,000 Syrian refugees have arrived in Germany to take shelter since the years 2010/2011, which marked the eruption of the Syrian civil war [1]. The majority of Syrian refugees were exposed to a variety of potentially traumatizing events, such as military combat, torture or imprisonment. Regardless whether such events were witnessed or personally experienced, they may increase 
vulnerability to post-traumatic stress and related adverse mental health outcomes [2]. A study reported that $75.3 \%$ among a group of 518 adult Syrian refugees in Germany had witnessed and/or experienced traumatic events. Subsequently, symptoms of post-traumatic stress disorder (PTSD) were reported by $11.4 \%$ of them [2]. Additionally, PTSD is often associated with a range of comorbidities that further compromise mental health. The most common comorbidities are: mood disorders (such as depression or bipolar disorders), anxiety disorders (such as generalized anxiety disorder, panic disorder), substance dependence, somatization disorders and increased attempts of suicide [3-5]. For example, moderate to severe depression was present in $14.5 \%$ and mild to severe generalized anxiety in $13.5 \%$ among Syrian adult refugees [2]. PTSD can furthermore result in distress or impaired social functioning [6-8]. Traumatic experiences tend to be stored in the implicit memory, but often only in parts, which makes a holistic narrative impossible. This frequently leads to intrusions and avoidance behaviour. In addition to post-traumatic stress, problems with residential status in the host country can further negatively impact mental health [9]. Therefore, psychotherapeutic interventions are highly indicated, and it has been recommended that they should be offered promptly after the arrival of refugees $[10,11]$. If treatment is not available, symptoms of PTSD and depression may be present in refugees even 20 years after their escape [12].

Indeed, there is a lack of adequate treatment possibilities due to a lack of sufficient treatment centres that offer psychological help or psychotherapy for refugees in host countries. This is mainly due to intercultural and language barriers that hinder utilization of help. Therefore, e-health options such as mobile phone-based interventions may be a possibility to fill the gap. This was the aim of our project Help@App. We developed a low-threshold self-help app "Sanadak" in Arabic language that is built on evidence-based cognitive-behavioural therapy (CBT) for PTSD. The content of the "Sanadak" app is multi-modal, i.e., it includes psychoeducational information to increase knowledge and awareness of PTSD and related mental health issues, and self-help techniques as well as skills training with respect to symptom management. In addition, a short self-test on post-traumatic symptom severity is implemented to allow for automated tailored feedback regarding progress at any time. Interactive materials, such as animated videos and audios as well as games and exercises are provided to maximize usability. The effectiveness of the app is evaluated in a randomized-controlled trial (RCT) with two arms: the intervention group uses the app and the control group receives psychoeducational reading material in order to increase knowledge and awareness of PTSD. Details have been described elsewhere [13].

The aim of this report was to describe (1) the recruitment processes including details on drop-out and eligibility for randomization as well as recruitment sample characteristics and (2) baseline characteristics of study participants with regard to group allocation (intervention and control group).

\section{Materials and Methods}

\subsection{Recruitment and Recruitment Procedures}

Recruitment and eligibility screening began in October 2018 in Leipzig, Germany. Participants were recruited in a multi-modal manner, as described elsewhere in detail [14]. Recruitment methods included, among others, snowball sampling, personal contacts of the study personnel who were conducting recruitment and interviews as well as cooperation with multipliers, i.e., facilities that actively work with the target group. Comprehensive study material in Arabic language was developed and used to further attract new prospects and to inform about the study. In addition, a broad range of contact possibilities (i.e., WhatsApp, Telegram, email, telephone contact, and social media) was offered.

Individuals who signaled interest in taking part in the study were screened to assess eligibility for trial participation with regard to post-traumatic stress symptom severity, symptoms of depression, and suicidal risk in accordance with the study protocol [13]. Inclusion criteria were: Syrian refugee living in Germany, aged 18-65 years, the experience of at least one traumatic event and subsequent mild to moderate post-traumatic stress symptom severity (Posttraumatic Diagnostic Scale for DSM-5, 
PDS-5 $=11-59$ [15]) and owning a compatible device in order to be able to use the app (Android/iOS). Moreover, literacy was a requirement. Exclusion criteria included post-traumatic stress symptomatology outside of the range mentioned above, severe depressive symptoms (Patient Health Questionnaire, PHQ-9 $\geq 20$ [16]), acute suicidal tendencies (Depressive Symptom Inventory-Suicidality Subscale, DSI-SS $\geq 3$ [17]), current psychotherapy/psychiatric treatment and/or psychotropic medication as well as pregnancy. If individuals were not eligible for trial participation due to severity of symptoms, they received psychoeducational material on mental health care and contact information of regional initiatives that offer face-to-face support. In five cases, the DSI-SS suicidal score was slightly over the pre-defined cutoff score of 3 . Eligibility was still considered based on the overall clinical impression of each prospect; as all of them credibly demonstrated no intention to act. These decisions were reached in consensus conferences between study nurses and study psychologists. Stability in inclusion and exclusion criteria were monitored during baseline- and follow up-assessments to ensure compliance with the study protocol. The recruitment process and sample selection are summarized in Figure 1. Recruitment and eligibility screening processes were completed in December 2019.

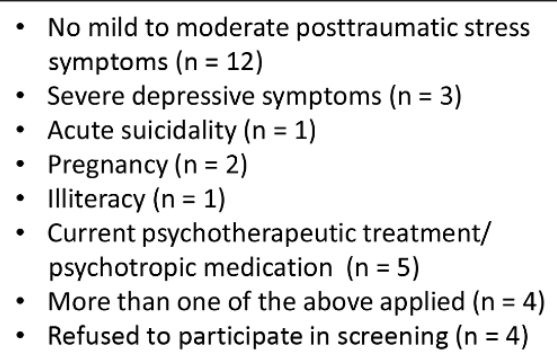

- Severe depressive symptoms $(n=3)$

- Acute suicidality $(n=1)$

- Pregnancy $(n=2)$

- Illiteracy $(n=1)$

- Current psychotherapeutic treatment/ psychotropic medication $(n=5)$

- More than one of the above applied $(n=4)$

- Refused to participate in screening $(n=4)$
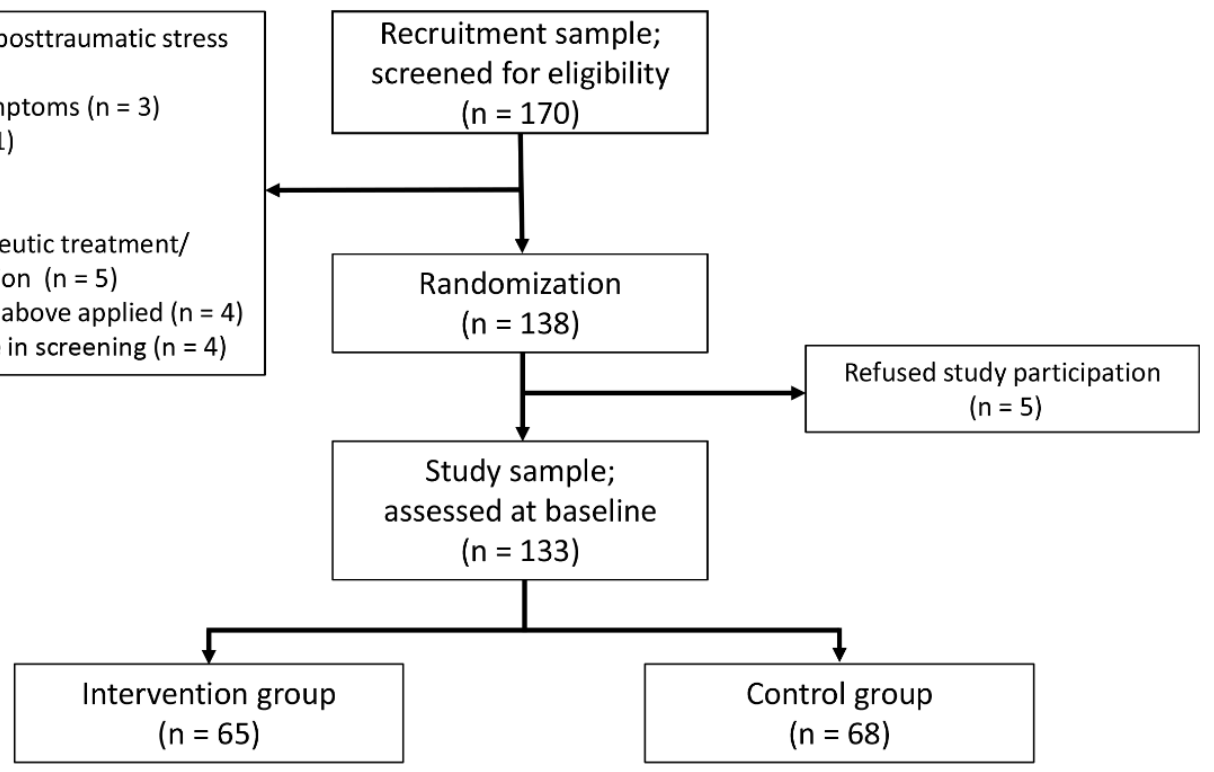

Figure 1. Flow chart of the recruitment process and sample selection.

\subsection{Randomization}

Participants were randomly allocated to the intervention or control group using a 1:1 ratio utilizing randomized permuted blocks of six, stratified by age and sex in order to ensure balance in sample size and distribution of covariates. The randomization block list was generated by an external, independent statistician using the "blockrand" package provided by the statistics software R. These block lists were then coded, leaving the person who was responsible for group allocation blind to the strata identity.

\subsection{Assessments and Instruments}

Overall, the study included up to four assessments (screening, baseline, follow-up 1 and 2). During each assessment, trained study nurses interviewed a participant using a written, structured questionnaire (paper-and-pencil assessment, face-to-face). The first assessment was the eligibility screening, outlined above, including questions on sociodemographic characteristics, information related to the escape, and instruments for measuring eligibility in accordance to mental health. In addition to the measures described in the Recruitment section above, resilience was assessed using the Resilience Scale (RS-13, [18]) during screening. 
Sociodemographic characteristics included information on age, gender, net personal income, living situation, residence status, religious group and religiosity (Centrality of Religiosity Scale/CRS, [19]). Information on education was gathered based on scholastic and professional qualifications with regard to the Syrian educational system. We then categorized the level of education (low, medium, high) based on the Comparative Analysis of Social Mobility in Industrial Nations (CASMIN) educational classification system [20].

After the screening, eligible individuals, who agreed to take part in the trial, underwent an additional comprehensive assessment on psychosocial health and associated factors (baseline assessment), including, among others, measures on generalized anxiety (GAD-7, [21]), severity of somatic symptoms (PHQ-15, [22]), general self-efficacy (GSE, [23]); self-stigma (Self-stigma of Mental Illness Scale-Short Form, SSMIS-SF, [24]); social support (short form of the Lubben Social Network Scale, LSNS-6, [25]; ENRICHD Social Support Inventory, ESSI, [26]); and health-related quality of life and health status (5-level version of EQ-5D and EQVAS, EQ-5D-5L, [27]). Please see the aforementioned study protocol for further information [13].

\subsection{Data Entry and Data Quality Control}

Data entry took place immediately after data collection using the statistical software SPSS 24 (IBM Corp., Armonk, NY, USA). Concurrently, data completeness and consistency checks were conducted to ensure data integrity. With regard to escape-related information, three participants refused to give answers. In addition, another three participants refused to give an answer on the item asessing "pain or problems during sexual intercourse" of the PHQ-15. Missing values were replaced with the item's mean score of all available responses [28]. The database is stored locally and only study personnel who have signed data protection wavers are able to access it. Each participant was given an ID; therefore, pseudonymity was ensured.

\subsection{Ethics and Registration}

The study was approved by the Ethics committee of the Medical Faculty of the University of Leipzig, Germany (ID: 111-17-ek) and adheres to the Declaration of Helsinki. All participants were informed about the study aims, including clarification about data security according to latest legal standards. Participation was only allowed after written informed consent. The study was pre-registered at the German Clinical Trials Register/Deutsches Register Klinischer Studien (DRKS; registration ID: DRKS00013782; date: 6th of July 2018).

\subsection{Statistical Analyses}

Group differences in the recruitment sample (randomized vs. nonrandomized) and in the study sample (intervention group vs. control group) were inspected using Chi-squared tests and $t$-tests, as appropriate. Analyses were conducted using Stata 16 (SE; StataCorp., College Station, TX, USA).

\section{Results}

\subsection{Recruitment Sample Characteristics}

Results with regard to the recruitment sample are summarized in Table 1 (sociodemographic characteristics), Table 2 (escape-related information, traumatic events and post-traumatic stress symptoms) and Table 3 (secondary outcomes). 
Table 1. Sociodemographic characteristics of the recruitment sample screened for eligibility in the "Sanadak" trial with regard to randomization status.

\begin{tabular}{|c|c|c|c|c|}
\hline \multirow{2}{*}{ Variables } & $\begin{array}{l}\text { Recruitment } \\
\text { Sample }\end{array}$ & Randomized $^{1}$ & Non-Randomized ${ }^{1}$ & \multirow{2}{*}{$p$-Value } \\
\hline & $(n=170)$ & $(n=133)$ & $(n=32)$ & \\
\hline Age (M, SD) & Ø 32.8 (11.2) & $\varnothing 33.3$ (11.2) & Ø 30.4 (11.8) & 0.19 \\
\hline \multicolumn{5}{|l|}{ Gender } \\
\hline Male & $104(61.2 \%)$ & $82(61.6 \%)$ & $19(59.4 \%)$ & \\
\hline Female & $66(38.8 \%)$ & $51(38.3 \%)$ & $13(40.6 \%)$ & 0.812 \\
\hline \multicolumn{5}{|l|}{$\begin{array}{c}\text { Net personal } \\
\text { income (monthly) }\end{array}$} \\
\hline$<500 €$ & $35(20.6 \%)$ & $26(19.5 \%)$ & $8(25.0 \%)$ & \\
\hline $500-999 €$ & $90(52.9 \%)$ & $70(52.6 \%)$ & $19(59.4 \%)$ & \\
\hline $1000-1499 €$ & $20(11.8 \%)$ & $15(11.3 \%)$ & $5(15.6 \%)$ & \\
\hline $1500-1999 €$ & $12(7.1 \%)$ & $10(7.5 \%)$ & 0 & \\
\hline $2000-2499 €$ & $6(3.5 \%)$ & $6(4.5 \%)$ & 0 & \\
\hline $2500-2999 €$ & $2(1.2 \%)$ & $2(1.5 \%)$ & 0 & \\
\hline not specified & $5(2.9 \%)$ & $4(3.0 \%)$ & 0 & 0.392 \\
\hline \multicolumn{5}{|l|}{$\begin{array}{l}\text { Education (school } \\
\text { based) }\end{array}$} \\
\hline$<12$ years & $53(31.2 \%)$ & $37(27.8 \%)$ & $11(35.5 \%)$ & \\
\hline$\geq 12$ years & $113(66.5 \%)$ & $94(70.7 \%)$ & $19(61.3 \%)$ & \\
\hline not specified & $4(2.3 \%)$ & $2(1.5 \%)$ & $2(6.2 \%)$ & 0.54 \\
\hline \multicolumn{5}{|l|}{$\begin{array}{l}\text { Educational level } \\
\text { (CASMIN) }\end{array}$} \\
\hline Low & $34(24.3 \%)$ & $25(22.9 \%)$ & $7(26.9 \%)$ & \\
\hline Middle & $58(41.4 \%)$ & $44(40.4 \%)$ & $14(53.8 \%)$ & \\
\hline High & $48(34.3 \%)$ & $40(36.7 \%)$ & $5(19.2 \%)$ & 0.229 \\
\hline \multicolumn{5}{|l|}{ Work permit } \\
\hline Yes & $151(88.8 \%)$ & $117(88.0 \%)$ & $29(90.6 \%)$ & \\
\hline No & $12(7.1 \%)$ & $11(8.3 \%)$ & $1(3.1 \%)$ & \\
\hline $\begin{array}{l}\text { Not specified } \\
\text { Status of } \\
\text { employment }^{2}\end{array}$ & $10(5.9 \%)$ & $5(3.7 \%)$ & $2(6.2 \%)$ & 0.512 \\
\hline Not employed & $117(68.8 \%)$ & $90(67.7 \%)$ & $24(75.0 \%)$ & \\
\hline $\begin{array}{l}\text { Marginally } \\
\text { employed }\end{array}$ & $24(14.1 \%)$ & $20(15.0 \%)$ & $4(12.5 \%)$ & \\
\hline $\begin{array}{l}\text { Part-time } \\
\text { employment }\end{array}$ & $15(8.8 \%)$ & $13(9.8 \%)$ & $2(6.2 \%)$ & \\
\hline $\begin{array}{l}\text { Full-time } \\
\text { employment }\end{array}$ & $12(7.1 \%)$ & $8(6.0 \%)$ & $2(6.2 \%)$ & \\
\hline $\begin{array}{c}\text { Not specified } \\
\text { Status of } \\
\text { unemployment }^{2}\end{array}$ & $2(1.2 \%)$ & $2(1.5 \%)$ & / & 0.886 \\
\hline $\begin{array}{c}\text { Integration } \\
\text { program }\end{array}$ & $7(5.7 \%)$ & $6(6.2 \%)$ & $1(4.3 \%)$ & \\
\hline $\begin{array}{c}\text { Federal Voluntary } \\
\text { Service }\end{array}$ & $5(4.1 \%)$ & $4(4.1 \%)$ & $1(4.3 \%)$ & \\
\hline Apprentice & $4(3.2 \%)$ & $4(4.1 \%)$ & l & \\
\hline $\begin{array}{l}\text { Labour market } \\
\text { (re-)training }\end{array}$ & $1(0.8 \%)$ & $1(1.0 \%)$ & l & \\
\hline Language course & $16(13.0 \%)$ & $11(11.3 \%)$ & $5(21.7 \%)$ & \\
\hline $\begin{array}{l}\text { Solely } \\
\text { housekeeping }\end{array}$ & $14(11.4 \%)$ & $13(13.4 \%)$ & $1(4.3 \%)$ & \\
\hline Student & $43(35.0 \%)$ & $33(34.0 \%)$ & $8(34.8 \%)$ & \\
\hline $\begin{array}{l}\text { Registered as } \\
\text { unemployed }\end{array}$ & $27(22.0 \%)$ & $23(23.7 \%)$ & $4(17.4 \%)$ & \\
\hline Not specified & $6(4.9 \%)$ & $2(2.1 \%)$ & $3(13.0 \%)$ & 0.278 \\
\hline
\end{tabular}


Table 1. Cont.

\begin{tabular}{|c|c|c|c|c|}
\hline \multirow{2}{*}{ Variables } & $\begin{array}{c}\text { Recruitment } \\
\text { Sample }\end{array}$ & Randomized $^{1}$ & Non-Randomized ${ }^{1}$ & \multirow[t]{2}{*}{$p$-Value } \\
\hline & $(n=170)$ & $(n=133)$ & $(n=32)$ & \\
\hline \multicolumn{5}{|l|}{ Living situation } \\
\hline Alone & $38(22.3 \%)$ & $33(24.8 \%)$ & $4(12.5 \%)$ & \\
\hline $\begin{array}{c}\text { With } \\
\text { family/relatives }\end{array}$ & $98(57.6 \%)$ & $73(54.9 \%)$ & $22(68.7 \%)$ & \\
\hline $\begin{array}{l}\text { With other people } \\
\text { (private home) }\end{array}$ & $31(18.2 \%)$ & $25(18.8 \%)$ & $5(15.6 \%)$ & \\
\hline $\begin{array}{l}\text { Communal } \\
\text { accommodation }\end{array}$ & $3(1.8 \%)$ & $2(1.5 \%)$ & $1(3.1 \%)$ & 0.373 \\
\hline \multicolumn{5}{|l|}{ Family status } \\
\hline Single & $83(48.8 \%)$ & $69(51.9 \%)$ & $11(34.4 \%)$ & \\
\hline Married & $69(40.6 \%)$ & $51(38.3 \%)$ & $16(50.0 \%)$ & \\
\hline Divorced & $10(5.9 \%)$ & $7(5.3 \%)$ & $3(9.4 \%)$ & \\
\hline Widowed & $3(1.8 \%)$ & $3(2.3 \%)$ & 0 & \\
\hline Not specified & $5(2.9 \%)$ & $3(2.3 \%)$ & $2(6.2 \%)$ & 0.259 \\
\hline \multicolumn{5}{|l|}{ Residence Status ${ }^{3}$} \\
\hline Asylum applicant & $21(12.3 \%)$ & $15(11.3 \%)$ & $4(12.5 \%)$ & \\
\hline \multicolumn{5}{|l|}{ Residence permit: } \\
\hline Refugee status & $74(43.5 \%)$ & $63(47.4 \%)$ & $10(31.2 \%)$ & \\
\hline $\begin{array}{l}\text { Subsidiary } \\
\text { protection }\end{array}$ & $14(8.2 \%)$ & $11(8.3 \%)$ & $3(9.4 \%)$ & \\
\hline $\begin{array}{l}\text { Humanitarian } \\
\text { protection }\end{array}$ & $40(23.5 \%)$ & $27(20.3 \%)$ & $12(37.5 \%)$ & \\
\hline Not specified & $21(12.3 \%)$ & $17(12.8 \%)$ & $3(9.4 \%)$ & 0.408 \\
\hline
\end{tabular}

${ }^{1}$ randomized = eligible for study participation, study group allocation was performed; nonrandomized: prospect was not eligible for study participation after screening for inclusion and exclusion criteria; ${ }^{2}$ multiple answers possible, such as "marginally employed" and "student"; ${ }^{3}$ according to the Federal Office for Migration and Refugees [29], asylum seekers have successfully completed the application procedures, asylum applicants are still in the process, being provided temporary residence status. $\varnothing=$ mean score.

Table 2. Escape- and trauma-related characteristics of the recruitment sample screened for eligibility in the "Sanadak" trial with regard to randomization status.

\begin{tabular}{|c|c|c|c|c|}
\hline Variables & $\begin{array}{c}\text { Recruitment } \\
\text { Sample }(n=170)\end{array}$ & $\begin{array}{l}\text { Randomized }^{1} \\
\quad(n=133)\end{array}$ & $\begin{array}{l}\text { Non-Randomized }{ }^{1} \\
\quad(n=32)\end{array}$ & $p$-Value \\
\hline \multicolumn{5}{|l|}{ Escape route $^{2}$} \\
\hline By airplane & $76(44.7 \%)$ & $59(44.4 \%)$ & $15(46.9 \%)$ & \\
\hline Via land & $113(66.5 \%)$ & $86(64.7 \%)$ & $24(75.0 \%)$ & \\
\hline Via sea & $99(58.2 \%)$ & $76(57.1 \%)$ & $20(62.5 \%)$ & \\
\hline Via transit country & $31(18.2 \%)$ & $23(17.3 \%)$ & $7(21.9 \%)$ & 0.985 \\
\hline $\begin{array}{l}\text { Time since Syria } \\
\text { was left (month) }\end{array}$ & Ø 49.8 (18.7) & Ø 50.4 (19.3) & $\varnothing 47.2$ (16.9) & 0.39 \\
\hline $\begin{array}{l}\text { Time spent in } \\
\text { Germany (month) }\end{array}$ & $\varnothing 41.0(13.3)$ & $\varnothing 41.2$ (13.9) & Ø 38.9 (10.8) & 0.383 \\
\hline $\begin{array}{c}\text { Traumatic event } \\
\text { (PDS-5) } \\
\text { Serious, }\end{array}$ & & & & \\
\hline $\begin{array}{l}\text { life-threatening } \\
\text { illness }\end{array}$ & $7(4.1 \%)$ & $5(3.8 \%)$ & $2(6.2 \%)$ & \\
\hline Physical assault & $11(6.5 \%)$ & $9(6.8 \%)$ & $1(3.1 \%)$ & \\
\hline Sexual assault & $2(1.2 \%)$ & 0 & $2(6.2 \%)$ & \\
\hline Military/combat-related & $56(32.9 \%)$ & $47(35.3 \%)$ & $7(21.9 \%)$ & \\
\hline Child abuse & $3(1.8 \%)$ & $3(2.3 \%)$ & 0 & \\
\hline Accident & $4(2.3 \%)$ & $3(2.3 \%)$ & $1(3.1 \%)$ & \\
\hline Torture/Imprisonment & $14(8.2 \%)$ & $10(7.5 \%)$ & $4(12.5 \%)$ & \\
\hline
\end{tabular}


Table 2. Cont.

\begin{tabular}{|c|c|c|c|c|}
\hline Variables & $\begin{array}{c}\text { Recruitment } \\
\text { Sample }(n=170)\end{array}$ & $\begin{array}{c}\text { Randomized }^{1} \\
(n=133)\end{array}$ & $\begin{array}{c}\text { Non-Randomized } \\
\quad(n=32)\end{array}$ & $p$-Value \\
\hline Other ${ }^{3}$ & $66(38.8 \%)$ & $54(40.6 \%)$ & $11(34.4 \%)$ & \\
\hline $\begin{array}{l}\text { Not further } \\
\text { specified }\end{array}$ & $7(4.1 \%)$ & $2(1.5 \%)$ & $4(12.5 \%)$ & $0.007, \mathrm{~V}=0.357$ \\
\hline $\begin{array}{l}\text { Posttraumatic } \\
\text { stress symptoms } \\
\quad\left(\text { PDS-5) }{ }^{2}\right.\end{array}$ & Ø 23.6 (13.2) & Ø 24.4 (11.1) & Ø 20.3 (20.5) 1 & 0.141 \\
\hline Intrusion & $5.5(4.1)$ & $5.6(3.8)$ & $4.5(5.1)$ & 0.177 \\
\hline Avoidance & $2.6(2.2)$ & $2.6(2.2)$ & $2.6(2.4)$ & 0.883 \\
\hline $\begin{array}{c}\text { Change in } \\
\text { Cognition \& Mood }\end{array}$ & $8.5(5.9)$ & $8.7(5.4)$ & $7.4(8.1)$ & 0.297 \\
\hline $\begin{array}{c}\text { Arousal \& } \\
\text { Hyperactivity }\end{array}$ & $7.1(4.4)$ & $7.4(3.8)$ & $6.0(6.5)$ & 0.11 \\
\hline
\end{tabular}

Note: $\varnothing=$ mean score; PDS-5 = Posttraumatic Diagnostic Scale for DSM-5; V = Cramér's V effect size; ${ }^{1}$ randomized = eligible for study participation, study group allocation was performed; nonrandomized: prospect was not eligible for study participation after screening for inclusion and exclusion criteria; ${ }^{2}$ missing data: $n=3$, not included; ${ }^{3}$ in most cases specified as escape-related events.

Table 3. Secondary mental health characteristics of the recruitment sample screened for eligibility in the "Sanadak" trial with regard to randomization status.

\begin{tabular}{|c|c|c|c|c|}
\hline \multirow[t]{2}{*}{ Variables } & $\begin{array}{c}\text { Recruitment } \\
\text { Sample }\end{array}$ & \multirow{2}{*}{$\begin{array}{l}\text { Randomized }^{1} \\
\quad(n=133)\end{array}$} & \multirow{2}{*}{$\begin{array}{c}\text { Non-Randomized } \\
\quad(n=32)\end{array}$} & \multirow[t]{2}{*}{$p$-Value } \\
\hline & $(n=170)$ & & & \\
\hline $\begin{array}{c}\text { Depressive } \\
\text { symptoms (PHQ-9) }\end{array}$ & Ø $9.4(5.8)$ & Ø $9.4(5.1)$ & Ø 9.5 (8.2) & 0.956 \\
\hline $\begin{array}{l}\text { No/low symptom } \\
\text { severity }(<10)\end{array}$ & $91(53.5 \%)$ & $70(52.6 \%)$ & $19(59.4 \%)$ & \\
\hline $\begin{array}{l}\text { Clinical significant } \\
\text { severity }(\geq 10)\end{array}$ & $79(46.5 \%)$ & $63(47.4 \%)$ & $13(40.6 \%)$ & 0.492 \\
\hline Resilience (RS-13) & Ø 64.3 (12.7) & Ø 64.4 (11.4) & Ø $63.1(17.0)$ & 0.615 \\
\hline Low (13-66) & $88(51.8 \%)$ & $70(52.6 \%)$ & $16(50.0 \%)$ & \\
\hline Middle (67-72) & $32(18.8 \%)$ & $25(18.8 \%)$ & $6(18.7 \%)$ & \\
\hline High (73-91) & $50(29.4 \%)$ & $38(28.6 \%)$ & $10(31.2 \%)$ & 0.952 \\
\hline $\begin{array}{l}\text { Suicidal risk } \\
\text { (DSI-SS) }\end{array}$ & $\varnothing 0.4(1.3)$ & $\varnothing 0.2(1.0)$ & $\varnothing 1.0(2.1)$ & $\begin{array}{c}0.002 \\
\mathrm{~d}=-0.623\end{array}$ \\
\hline $\begin{array}{l}\text { No suicidal risk } \\
\qquad(<3)\end{array}$ & $160(94.1 \%)$ & $128(96.2 \%)$ & $26(81.2 \%)$ & 0.002 \\
\hline $\begin{array}{l}\text { Elevated suicidal } \\
\text { risk }(\geq 3)\end{array}$ & $10(5.9 \%)$ & $5(3.8 \%)$ & $6(18.7 \%)$ & $\mathrm{V}=0.238$ \\
\hline
\end{tabular}

Note: $\varnothing=$ mean score; PHQ-9 = Patient Health Questionnaire; RS-13 = Resilience Scale; DSI-SS = Depressive Symptom Inventory-Suicidality Subscale, $\mathrm{d}=$ Cohen's $\mathrm{d}$ effect size; $\mathrm{V}=$ Cramér's $\mathrm{V}$ effect size; ${ }^{1}$ randomized $=$ eligible for study participation, study group allocation was performed; nonrandomized: prospect was not eligible for study participation after screening for inclusion and exclusion criteria.

\subsubsection{Sociodemographic Characteristics of the Recruitment Sample}

The mean age of the screened participants was $32.8(S D=11.2)$ years and the majority was male $(61.2 \%)$ (Table 1). Overall, $66.5 \%$ went to school for 12 or more years. In terms of net personal income, more than $73 \%$ of participants indicated that their current income was less than 1000 Euros per month. Concerning the living situation and family status, $57.6 \%$ of participants were currently living with family members or relatives; $48.8 \%$ were single and $40.6 \%$ were married. Regarding their residence status, $12.3 \%$ of the screening participants were "asylum seeker", $43.5 \%$ were entitled to asylum and $23.5 \%$ fell under the "refugee protection status". Though the majority had a working permit $(88.8 \%)$, about two-thirds reported to be unemployed (68.8\%); however, $35.0 \%$ of those were students. 
Five eligible individuals refused further participation after randomization.

\subsubsection{Escape- and Trauma-Related Characteristics of the Recruitment Sample}

Findings with regard to escape-related information, traumatic events and post-traumatic stress symptoms are summarized in Table 2 . The majority of participants escaped via land $(66.5 \%)$ and sea (58.2\%); followed by airplanes (44.7\%), and $18.2 \%$ stated that they escaped via transit countries. On average, participants had left Syria more than four years ago (49.8 months) and have spent about three and a half years (41 months) in Germany.

Most frequently experienced traumatic events were military or combat-related events $(32.9 \%)$ and "others" (38.8\%), which were mostly specified as escape-related events. In two cases, randomized participants were not willing to specify their most stressful traumatic event. However, they were referring to the nonspecified event when answering questions on the PDS-5-scale. The total PDS5-score was $M=23.6$ ( $S D=13.2$; randomized group: $M=24.4, S D=11.1$; nonrandomized group: $M=20.3, S D=20.5 ; p=133)$. The five participants that dropped out after being randomized had a mean PDS-5-score of $23.8(S D=8.5)$.

\subsubsection{Secondary Mental Health Characteristics of the Recruitment Sample}

Secondary outcomes, including depressive symptoms, resilience and suicidal risk are summarized in Table 3. The overall PHQ-9 score indicating depressive symptoms was $9.4(S D=5.8)$ on average. Assuming major depression with a cut-off value of 10 or more, $46.5 \%$ of the recruitment sample had major depression ( $47.7 \%$ in the randomized group and $40.6 \%$ in the nonrandomized group; $p=0.492)[16]$.

The resilience score, measured using the RS-13, was $64.3(S D=12.7)$ on average, which is considered low resilience. Results of the Depressive Symptom Inventory-Suicidality Subscale (DSI-SS) showed a mean score of $0.4(S D=1.3)$, with $0.2(S D=1.0)$ in the randomized group and $1.0(S D=2.1)$ in the nonrandomized group $(p<0.05)$. Higher scores in the nonrandomized group are due to the exclusion of elevated suicidal risk.

\subsection{Study Sample Characteristics}

After successful screening, 133 study participants were randomized into the intervention group $(n=65)$ or control group $(n=68)$. Results with regard to the study sample are described in Table 4 (sociodemographic characteristics), Table 5 (escape-related information, traumatic events and posttraumatic stress symptoms) and Table 6 (secondary outcomes). There were no significant group differences with regard to sociodemographic characteristics.

Table 4. Sociodemographic characteristics of the "Sanadak" study sample with regard to group allocation.

\begin{tabular}{ccccc}
\hline Variables & Study Sample & $\begin{array}{c}\text { Intervention } \\
\text { Group }(\boldsymbol{n}=\mathbf{6 5})\end{array}$ & $\begin{array}{c}\text { Control Group } \\
(\boldsymbol{n}=\mathbf{6 8})\end{array}$ & $\boldsymbol{p}$-Value \\
\cline { 2 - 3 } Age $(\mathrm{M}, \mathrm{SD})$ & $\varnothing 33.3(11.2)$ & $\varnothing 33.0(11.0)$ & $\varnothing 33.7(11.4)$ & 0.723 \\
Gender & $82(61.6 \%)$ & $43(66.2 \%)$ & $39(57.4 \%)$ & \\
Male & $51(38.3 \%)$ & $22(33.8 \%)$ & $29(42.6 \%)$ & \\
Female & & & & \\
Net personal & $26(19.5 \%)$ & $13(20.0 \%)$ & $13(19.1 \%)$ & \\
income & $70(52.6 \%)$ & $32(49.2 \%)$ & $38(55.9 \%)$ & \\
$<500 €$ & $15(11.3 \%)$ & $7(10.8 \%)$ & $8(11.8 \%)$ & \\
$500-999 €$ & $10(7.5 \%)$ & $6(9.2 \%)$ & $4(5.9 \%)$ & \\
$1000-1499 €$ & $6(4.5 \%)$ & $5(7.7 \%)$ & $1(1.5 \%)$ & \\
$1500-1999 €$ & $2(1.5 \%)$ & 0 & $2(2.9 \%)$ & \\
$2000-2499 €$ & & & \\
$2500-2999 €$ & &
\end{tabular}


Table 4. Cont.

\begin{tabular}{|c|c|c|c|c|}
\hline Variables & $\begin{array}{c}\text { Study Sample } \\
(n=133)\end{array}$ & $\begin{array}{l}\text { Intervention } \\
\text { Group }(n=65)\end{array}$ & $\begin{array}{l}\text { Control Group } \\
\quad(n=68)\end{array}$ & $p$-Value \\
\hline $\begin{array}{c}\text { not specified } \\
\text { Education } \\
\text { (school-based) }\end{array}$ & $4(3.0 \%)$ & $2(3.1 \%)$ & $2(2.9 \%)$ & 0.472 \\
\hline$<12$ years & $37(27.8 \%)$ & $16(24.6 \%)$ & $21(30.9 \%)$ & \\
\hline$\geq 12$ years & $94(70.7 \%)$ & $47(72.3 \%)$ & $47(69.1 \%)$ & \\
\hline No school visit & $2(1.5 \%)$ & $2(3.1 \%)$ & 0 & 0.271 \\
\hline \multicolumn{5}{|l|}{$\begin{array}{c}\text { Educational level } \\
\text { (CASMIN) }\end{array}$} \\
\hline Low & $25(22.9 \%)$ & $12(24.0 \%)$ & $13(22.0 \%)$ & \\
\hline Middle & $44(40.4 \%)$ & $18(36.0 \%)$ & $26(44.1 \%)$ & \\
\hline High & $40(36.7 \%)$ & $20(40.0 \%)$ & $20(33.9 \%)$ & 0.685 \\
\hline \multicolumn{5}{|l|}{ Work permit } \\
\hline Yes & 117 (88.0\%) & $57(87.7 \%)$ & $60(88.2 \%)$ & \\
\hline No & $11(8.3 \%)$ & $6(9.2 \%)$ & $5(7.3 \%)$ & \\
\hline $\begin{array}{l}\text { Not specified } \\
\text { Status of } \\
\text { employment }\end{array}$ & $5(3.7 \%)$ & $2(3.1 \%)$ & $3(4.4 \%)$ & 0.933 \\
\hline Not employed & $90(67.7 \%)$ & $40(61.5 \%)$ & $50(73.5 \%)$ & \\
\hline $\begin{array}{l}\text { Marginally } \\
\text { employed }\end{array}$ & $20(15.0 \%)$ & $10(15.4 \%)$ & $10(14.7 \%)$ & \\
\hline $\begin{array}{l}\text { Part-time } \\
\text { employment }\end{array}$ & $13(9.8 \%)$ & $6(9.2 \%)$ & $7(10.3 \%)$ & \\
\hline $\begin{array}{l}\text { Full-time } \\
\text { employment }\end{array}$ & $8(6.0 \%)$ & $8(12.3 \%)$ & / & \\
\hline $\begin{array}{l}\text { Not specified } \\
\text { Status of } \\
\text { unemployment }\end{array}$ & $2(1.5 \%)$ & $1(1.5 \%)$ & $1(1.5 \%)$ & 0.058 \\
\hline $\begin{array}{l}\text { Integration } \\
\text { program }\end{array}$ & $6(6.2 \%)$ & $2(4.8 \%)$ & $4(7.1 \%)$ & \\
\hline $\begin{array}{c}\text { Federal Voluntary } \\
\text { Service }\end{array}$ & $4(4.1 \%)$ & $1(2.4 \%)$ & $3(5.4 \%)$ & \\
\hline Apprentice & $4(4.1 \%)$ & $3(7.1 \%)$ & $1(1.8 \%)$ & \\
\hline $\begin{array}{l}\text { Labour market } \\
\text { (re-)training }\end{array}$ & $1(1.0 \%)$ & / & $1(1.8 \%)$ & \\
\hline Language course & $11(11.3 \%)$ & $7(16.7 \%)$ & $4(7.1 \%)$ & \\
\hline $\begin{array}{c}\text { Solely } \\
\text { housekeeping }\end{array}$ & $13(13.4 \%)$ & $6(14.3 \%)$ & 7 (12.5\%) & \\
\hline Student & $33(34.0 \%)$ & $12(28.6 \%)$ & $21(37.5 \%)$ & \\
\hline $\begin{array}{l}\text { Registered as } \\
\text { unemployed }\end{array}$ & $23(23.7 \%)$ & $8(19.0 \%)$ & $15(26.8 \%)$ & \\
\hline Not specified & $3(3.1 \%)$ & $3(7.1 \%)$ & l & 0.322 \\
\hline Living situation & & & & \\
\hline Alone & $33(24.8 \%)$ & $18(27.7 \%)$ & $15(22.1 \%)$ & \\
\hline $\begin{array}{c}\text { With } \\
\text { family/relatives }\end{array}$ & $73(54.9 \%)$ & $35(53.8 \%)$ & $38(55.9 \%)$ & \\
\hline $\begin{array}{l}\text { With other people } \\
\text { (private home) }\end{array}$ & $25(18.8 \%)$ & $12(18.5 \%)$ & $13(19.1 \%)$ & \\
\hline $\begin{array}{l}\text { Communal } \\
\text { accommodation }\end{array}$ & $2(1.5 \%)$ & 0 & $2(2.9 \%)$ & 0.718 \\
\hline Family status & & & & \\
\hline Single & $69(51.9 \%)$ & $37(56.9 \%)$ & $32(47.1 \%)$ & \\
\hline Married & $51(38.3 \%)$ & $21(32.3 \%)$ & $30(44.1 \%)$ & \\
\hline Divorced & $7(5.3 \%)$ & $4(6.1 \%)$ & $3(4.4 \%)$ & \\
\hline Widowed & $3(2.3 \%)$ & $2(3.1 \%)$ & $1(1.5 \%)$ & \\
\hline $\begin{array}{c}\text { Not specified } \\
\text { Residence status } 2\end{array}$ & $3(2.3 \%)$ & $1(1.5 \%)$ & $2(2.9 \%)$ & 0.61 \\
\hline
\end{tabular}


Table 4. Cont.

\begin{tabular}{|c|c|c|c|c|}
\hline Variables & $\begin{array}{c}\text { Study Sample } \\
(n=133)\end{array}$ & $\begin{array}{l}\text { Intervention } \\
\text { Group }(n=65)\end{array}$ & $\begin{array}{c}\text { Control Group } \\
\quad(n=68)\end{array}$ & $p$-Value \\
\hline Asylum applicant & $15(11.3 \%)$ & $7(10.8)$ & $8(11.8 \%)$ & \\
\hline \multicolumn{5}{|l|}{ Residence permit } \\
\hline Refugee status & $63(47.4 \%)$ & $31(47.7 \%)$ & $32(47.0 \%)$ & \\
\hline $\begin{array}{l}\text { Subsidiary } \\
\text { protection }\end{array}$ & $11(8.3 \%)$ & $5(7.7 \%)$ & $6(8.8 \%)$ & \\
\hline $\begin{array}{l}\text { Humanitarian } \\
\text { protection }\end{array}$ & $27(20.3 \%)$ & $12(18.5 \%)$ & $15(22.1 \%)$ & \\
\hline Not specified & $17(12.8 \%)$ & $10(15.4 \%)$ & $7(10.3 \%)$ & 0.945 \\
\hline $\begin{array}{l}\text { Religion and } \\
\text { religiosity }\end{array}$ & & & & \\
\hline \multicolumn{5}{|l|}{ Religious group } \\
\hline Muslim & $74(56.9 \%)$ & $38(56.9 \%)$ & $36(54.5 \%)$ & \\
\hline Sunnis & $51(68.9 \%)$ & $26(68.4 \%)$ & $25(69.4 \%)$ & \\
\hline Shiites & $2(2.7 \%)$ & $1(2.6 \%)$ & $1(2.8 \%)$ & \\
\hline Alawis & $4(5.4 \%)$ & $3(7.9 \%)$ & $1(2.8 \%)$ & \\
\hline $\begin{array}{l}\text { Not further } \\
\text { specified }\end{array}$ & $17(23.0 \%)$ & $8(21.1 \%)$ & $9(25.0 \%)$ & \\
\hline Christian & $9(6.9 \%)$ & $6(9.4 \%)$ & $3(4.5 \%)$ & \\
\hline Other & $12(9.2 \%)$ & $6(9.4 \%)$ & $6(9.4 \%)$ & \\
\hline None & $35(26.9 \%)$ & $14(10.8 \%)$ & $21(16.2 \%)$ & 0.71 \\
\hline Religiosity & $\varnothing 18.50(4.17)$ & Ø $18.51(3.89)$ & $\varnothing 18.49(4.46)$ & 0.983 \\
\hline Not religious $(<10)$ & $0(0 \%)$ & $0(0 \%)$ & $0(0 \%)$ & \\
\hline Religious (10-19) & $68(52.3 \%)$ & $38(58.5 \%)$ & $30(46.2 \%)$ & \\
\hline Very religious (>19) & $62(47.7 \%)$ & $27(41.5 \%)$ & $35(53.8 \%)$ & 0.16 \\
\hline
\end{tabular}

${ }^{1}$ Multiple answers possible, such as "marginally employed" and "student"; ${ }^{2}$ according to the Federal Office for Migration and Refugees [29], asylum seekers have successfully completed the application procedures, asylum applicants are still in the process, being provided temporary residence status. $\varnothing=$ mean score.

Table 5. Escape- and trauma-related characteristics of the "Sanadak" study sample with regard to group allocation.

\begin{tabular}{|c|c|c|c|c|}
\hline Variables & $\begin{array}{l}\text { Study Sample } \\
\quad(n=133)\end{array}$ & $\begin{array}{l}\text { Intervention } \\
\text { Group }(n=65)\end{array}$ & $\begin{array}{c}\text { Control Group } \\
\quad(n=68)\end{array}$ & $p$-Value \\
\hline \multicolumn{5}{|l|}{ Escape route $^{1}$} \\
\hline By airplane & $59(44.4 \%)$ & $25(38.5 \%)$ & $34(50.0 \%)$ & \\
\hline By land & $86(64.7 \%)$ & $43(66.1 \%)$ & $43(63.2 \%)$ & \\
\hline By sea & $76(57.1 \%)$ & $37(56.9 \%)$ & $39(57.3 \%)$ & \\
\hline Via transit country & $23(17.3 \%)$ & $13(20.0 \%)$ & $10(14.7 \%)$ & 0.669 \\
\hline $\begin{array}{c}\text { Years since Syria } \\
\text { was left (yrs) }\end{array}$ & Ø 50.4 (19.3) & Ø 3.9 (1.6) & $\varnothing 3.7$ (1.6) & 0.472 \\
\hline $\begin{array}{l}\text { Time spent in } \\
\text { Germany (yrs) }\end{array}$ & Ø 41.2 (13.9) & $\varnothing 3.0(1.1)$ & Ø $3.0(1.3)$ & 1 \\
\hline \multicolumn{5}{|l|}{$\begin{array}{c}\text { Traumatic event } \\
\text { (PDS-5) }\end{array}$} \\
\hline $\begin{array}{c}\text { Serious, } \\
\text { life-threatening } \\
\text { illness }\end{array}$ & $5(3.8 \%)$ & $4(6.1 \%)$ & $1(1.5 \%)$ & \\
\hline Physical assault & $9(6.8 \%)$ & $4(6.1 \%)$ & $5(7.3 \%)$ & \\
\hline Military/combat-related & $47(35.3 \%)$ & $21(32.3 \%)$ & $26(38.2 \%)$ & \\
\hline Child abuse & $3(2.3 \%)$ & $1(1.5 \%)$ & $2(2.9 \%)$ & \\
\hline Accident & $3(2.3 \%)$ & $2(3.1 \%)$ & $1(1.5 \%)$ & \\
\hline Torture/imprisonment & $10(7.5 \%)$ & $3(4.6 \%)$ & $7(10.3 \%)$ & \\
\hline
\end{tabular}


Table 5. Cont.

\begin{tabular}{ccccc}
\hline Variables & $\begin{array}{c}\text { Study Sample } \\
(n=133)\end{array}$ & $\begin{array}{c}\text { Intervention } \\
\text { Group }(n=65)\end{array}$ & $\begin{array}{c}\text { Control Group } \\
(n=68)\end{array}$ & $p$-Value \\
\hline $\begin{array}{c}\text { Other } \\
\text { Refusal of answer }\end{array}$ & $54(40.6 \%)$ & $29(44.6 \%)$ & $25(36.8 \%)$ & 0.667 \\
$\begin{array}{c}\text { Post-traumatic } \\
\text { stress symptoms }\end{array}$ & $\varnothing(1.5 \%)$ & $1(1.5 \%)$ & $1(1.5 \%)$ & 0.539 \\
$\begin{array}{c}\text { (PDS-5) } \\
\text { Intrusion }\end{array}$ & $5.6(4.1)$ & $\varnothing 23.2(10.8)$ & $\varnothing 24.4(12.4)$ & 0.483 \\
$\begin{array}{c}\text { Avoidance } \\
\text { Change in }\end{array}$ & $2.6(2.1)$ & $5.4(4.0)$ & $5.9(4.2)$ & 0.584 \\
$\begin{array}{c}\text { cognition \& mood } \\
\text { Arousal \& } \\
\text { hyperactivity }\end{array}$ & $8.4(5.4)$ & $2.5(1.9)$ & $2.7(2.2)$ & 0.439 \\
\hline
\end{tabular}

Note: $\varnothing=$ mean score; PDS-5 = Posttraumatic Diagnostic Scale for DSM-5.

Table 6. Secondary characteristics of the "Sanadak" study sample with regard to group allocation.

\begin{tabular}{|c|c|c|c|c|}
\hline Variables & $\begin{array}{c}\text { Study Sample } \\
(n=133)\end{array}$ & $\begin{array}{l}\text { Intervention } \\
\text { Group }(n=65)\end{array}$ & $\begin{array}{l}\text { Control Group } \\
\quad(n=68)\end{array}$ & $p$-Value \\
\hline Depressive symptoms (PHQ-9) & $\varnothing 9.2(5.2)$ & $\varnothing 9.2(4.8)$ & $\varnothing 9.3(5.7)$ & 0.84 \\
\hline No/low symptom severity $(<10)$ & $73(54.9 \%)$ & $37(56.9 \%)$ & $36(52.9 \%)$ & \\
\hline Clinical significant severity $(\geq 10)$ & $60(45.1 \%)$ & $28(43.1 \%)$ & $32(47.1 \%)$ & 0.645 \\
\hline Generalized anxiety (GAD-7) & $\varnothing 8.5(5.0)$ & $\varnothing 8.2(4.4)$ & $\varnothing 8.8(5.5)$ & 0.483 \\
\hline No/low symptom severity $(<10)$ & $79(59.4 \%)$ & $40(61.5 \%)$ & $39(57.3 \%)$ & \\
\hline Clinical significant severity $(\geq 10)$ & $54(40.6 \%)$ & $25(38.5 \%)$ & $29(42.6 \%)$ & 0.623 \\
\hline \multicolumn{5}{|l|}{ Somatization (PHQ-15) } \\
\hline Female & $\varnothing 10.5(5.3)$ & $\varnothing 10.0(5.2)$ & $\varnothing 11.1(5.4)$ & 0.441 \\
\hline Low symptom severity $(<10)$ & $18(35.3 \%)$ & $12(41.4 \%)$ & $6(27.3 \%)$ & \\
\hline $\begin{array}{l}\text { Medium-high symptom severity } \\
\qquad(\geq 10)\end{array}$ & $33(64.7 \%)$ & 17 (58.6\%) & $16(72.7 \%)$ & 0.296 \\
\hline Male & $\varnothing 7.8(5.1)$ & $\varnothing 7.7(5.0)$ & $\varnothing 7.9(5.3)$ & 0.853 \\
\hline Low symptom severity $(<10)$ & $52(63.4 \%)$ & $22(56.4 \%)$ & $30(69.8 \%)$ & \\
\hline $\begin{array}{l}\text { Medium-high symptom severity } \\
\qquad(\geq 10)\end{array}$ & $30(36.6 \%)$ & $17(43.6 \%)$ & $13(30.2 \%)$ & 0.21 \\
\hline Social network size (LSNS-6) & $\varnothing 15.1(5.3)$ & $\varnothing 15.0(5.5)$ & $\varnothing 15.2(5.2)$ & 0.785 \\
\hline Social isolation (LSNS-6 < 12) & $43(32.3 \%)$ & $21(32.3 \%)$ & $22(32.4 \%)$ & 0.996 \\
\hline Social support (ESSI) & $\varnothing 18.0(4.7)$ & $\varnothing 18.4(4.1)$ & $\varnothing 17.7$ (5.2) & 0.388 \\
\hline Low support & $71(53.4 \%)$ & $32(49.2 \%)$ & $39(57.4 \%)$ & \\
\hline High support & $62(46.6 \%)$ & $33(50.8 \%)$ & $29(42.6 \%)$ & 0.348 \\
\hline General self-efficacy (GSE) & $\varnothing 27.4(4.7)$ & $\varnothing 26.8(5.2)$ & $\varnothing 28.0(4.0)$ & 0.151 \\
\hline Resilience (RS-13) & $\varnothing 64.4(11.4)$ & $\varnothing 61.6(11.6)$ & $\varnothing 67.0(10.3)$ & $\begin{array}{c}0.006 \\
\mathrm{~d}=0.480\end{array}$ \\
\hline Low (13-66) & $70(52.6 \%)$ & $41(63.1 \%)$ & $29(42.6 \%)$ & \\
\hline Middle (67-72) & $25(18.8 \%)$ & $11(16.9 \%)$ & $14(20.6 \%)$ & 0.046 \\
\hline High (73-91) & $38(28.6 \%)$ & $13(20.0 \%)$ & $25(36.8 \%)$ & $\mathrm{V}=0.215$ \\
\hline \multicolumn{5}{|l|}{ Self-stigmatization (SSMIS-SF) } \\
\hline Awareness & $28.3(7.5)$ & $28.8(8.3)$ & $27.8(6.6)$ & 0.449 \\
\hline Agreement & $18.8(6.7)$ & $17.6(6.5)$ & $20.0(6.8)$ & $0.034, d=-0.372$ \\
\hline Application & $16.5(6.6)$ & $15.2(6.2)$ & $17.8(6.8)$ & $0.021, \mathrm{~d}=-0.404$ \\
\hline Harm to Self-esteem & $18.6(9.5)$ & $18.0(9.5)$ & $19.2(9.6)$ & 0.454 \\
\hline \multicolumn{5}{|l|}{$\begin{array}{l}\text { Health-related quality of life \& } \\
\text { subjective health }\end{array}$} \\
\hline EQ-5D-5L & $0.82(0.19)$ & $0.79(0.23)$ & $0.86(0.13)$ & 0.052 \\
\hline EQ-VAS & $73.6(18.9)$ & $73.0(20.7)$ & $74.2(16.9)$ & 0.713 \\
\hline
\end{tabular}


Table 6. Cont.

\begin{tabular}{|c|c|c|c|c|}
\hline Variables & $\begin{array}{c}\text { Study Sample } \\
(n=133)\end{array}$ & $\begin{array}{c}\text { Intervention } \\
\text { Group }(n=65)\end{array}$ & $\begin{array}{c}\text { Control Group } \\
(n=68)\end{array}$ & $p$-Value \\
\hline Suicidal risk (DSI-SS) & $\varnothing 0.2(1.0)$ & $\varnothing 0.03(0.2)$ & $\varnothing 0.2(1.1)$ & 0.168 \\
\hline$<3$ (no suicidal risk) & $128(96.2 \%)$ & $64(98.5 \%)$ & $65(95.6 \%)$ & \\
\hline$\geq 3$ (elevated suicidal risk) & $5(3.8 \%)$ & $1(1.5 \%)$ & $3(4.4 \%)$ & 0.268 \\
\hline
\end{tabular}

Note: $\varnothing=$ mean score; DSI-SS = Depressive Symptom Inventory-Suicidality Subscale; EQ-5D_5L = 5-level version of EQ-5D; EQ-VAS = EQ visual analogue scale; ESSI = ENRICHD Social Support Inventory; GAD-7 = Generalized Anxiety Disorder Scale-7; GSE = general self-efficacy; LSNS-6 = short form of the Lubben Social Network Scale; PHQ-9/-15 = Patient Health Questionnaire; RS-13 = Resilience Scale; SSMIS-SF = Self-Stigma of Mental Illness Scale-Short Form; $\mathrm{d}=$ Cohen's d effect size; $\mathrm{V}=$ Cramer's V effect size.

\subsubsection{Escape and Trauma-Related Characteristics of the "Sanadak" Study Sample}

Details on escape- and trauma-related characteristics are summarized in Table 5. Again, there were no significant differences between the intervention group and the control group.

\subsubsection{Secondary Mental Health Characteristics of the "Sanadak" Study Sample}

Secondary outcome measures at baseline are described in Table 6. Significant group differences between the intervention and control groups were found in regard to resilience and self-stigmatization. The overall RS-13 score was higher in the control group compared to the intervention group ( $M=67.0$, $S D=10.3$ vs. $M=61.6, S D=11.6 ; p<0.05$ ). In relation to stigmatization (SSMIS-SF scale), the control group showed significantly higher scores with regard to the subscale agreement $(M=20.0, S D=6.8 \mathrm{vs}$. $M=17.6, S D=6.5 ; p<0.05$ ). No group differences were found for other targeted variables.

\section{Discussion}

The aim of the project Help@App is to develop and evaluate the effectiveness of the interactive, Arabic language self-help app "Sanadak", which targets Syrian refugees with post-traumatic stress. Based on the results of the screening and baseline assessments, this report provides useful information before engaging in the RCT.

The main reason for noneligibility for trial participation after the screening was that the severity of post-traumatic stress symptoms did not meet the study's inclusion criteria. Out of 138 eligible individuals being randomized, five decided not to take part in the study and could therefore not be scheduled for baseline assessments. Finally, 133 individuals were allocated to the intervention group $(n=65)$ and to the control group $(n=68)$ and constituted the study sample of the "Sanadak" trial. Importantly, the randomization strategy proved to be successful as there were no group differences with regard to key sociodemographic and primary mental health outcomes after allocating eligible participants to the intervention group or control group. Furthermore, the presented results provide important key figures of a large, but not well-studied population group in Germany.

Previous studies investigating post-traumatic stress in refugees relied on a variety of different measures. Therefore, it is difficult to compare our results to other studies with a similar focus. The original study, that investigated the psychometric properties of the PDS- 5 relied on a broad sample of individuals with different ethnical backgrounds that had experienced a traumatic event according to the DSM- 5 criteria: the authors reported higher post-traumatic stress total scores in their sample compared to ours [15]. Further differences between the samples related to the type of traumas. Whereas physical assault, for example, was reported more frequently in the sample of Foa et al. [15] $(21.2 \%$ vs. $6.5 \%)$, military or combat-related trauma was much more often reported in our sample of Syrian refugees ( $12.6 \%$ vs. $32.9 \%)$.

In addition to post-traumatic stress, previous work on mental health in refugees and migrants mostly focused on depression and anxiety. Regarding depression, the majority of Syrian refugees reported increased depressive symptoms and over $46 \%$ of our recruitment sample had major depression. In a (nonrepresentative) survey with over 500 community-dwelling adult Syrian refugees, the PHQ-9 
depression mean score was slightly lower (7.1 compared to 9.2 in our study sample) [2]. However, a study with Syrian refugees living in housing facilities in Sweden reported a higher mean score (11.5) [30]. In an online survey by Euteneuer et al. [31], that comprised male Syrian refugees residing in Germany recruited via refugee information centers, the mean score was also higher (10.4) and $29 \%$ of the refugees classified for probable major depression. The aforementioned study by Georgiadou et al. [2] found moderate to severe generalized anxiety in $13.5 \%$ among their sample of community-dwelling adult Syrian refugees compared to over $40 \%$ in ours. Further analysis of somatic symptoms revealed higher symptom severity in female participants compared to male participants (PHQ-15 mean scores: 10.5 vs. 7.8), which was in line with previous study results [11], confirming gender-specific differences in somatisation in refugees. The number of individuals with acute suicidal tendencies in our sample was low. This is contrary to other studies that reported higher suicidality in refugees, including a population-based cohort [32] and compared to the general population [33-35]. However, as already stated before, the concept of suicidality may have lead to misunderstandings in our sample due to cultural differences. With regard to general self-efficacy, the mean score in our study sample was in good agreement with a study including Syrian participants (not described as refugees) [36]. We could not identify studies that adapted the resilience scale, RS-13, therefore, scores cannot be compared. However, with regard to the general population in Germany [18], the mean score in our sample was lower (64.3 vs. 70.0 ), therefore, the participants in our sample could be considered less resilient. However, our sample comprises individuals with relevant post-traumatic stress and high global psychological distress, which may be a result of lower resilience in the first place. The role of resilience requires further study. A significant difference was found with regard to the resilience mean scores in the intervention and control group. Participants allocated to the control group scored higher on the resilience scale. This difference will be addressed in the trial outcome analysis.

Social support, which has been described as a potential protective factor against PTSD, has also been a focus of this study. Less than half of our study participants reported high social support, which is comparable to a study that investigated social support in a random sample of Syrian refugees residing in Sweden [37]. Lastly, scores on self-stigma in relation to mental illness were in line with other studies, that used the same measure but focused on participants with serious mental health complaints without a refugee background [24].

\section{Limitations}

With regard to depressive symptoms as well as post-traumatic stress, we found slight deviations between the screening and the recruitment sample in regard to the randomized group (for PHQ-9: the mean score changed from 9.4 to 9.2; for PDS-5 the score changed from 24.4 to 23.8). This can be explained by the study procedure: In the majority of cases, baseline assessments were scheduled shortly after the screening assessments. However, in few cases, the time period between screening and baseline assessment exceeded two or more weeks due to difficulties in scheduling interviews with the participants, which required repeating the assessment of the PHQ-9 and PDS-5 in order to measure current states. Moreover, result comparisons with other studies investigating mental and social health outcomes in Syrian refugees have to be viewed with caution as sample selection tended to vary and samples were usually nonrandom. Lastly, even though assessments were very comprehensive (average duration: 2.5 to $3 \mathrm{~h}$ ), there may be other relevant factors, for example, perceived discrimination in the host country, that we were not able to cover due to time constraints.

\section{Conclusions}

The results of the recruitment and randomization procedures proved the allocation strategy to be successful, since distinctive characteristics with regard to sociodemographic information did not vary between targeted groups. Furthermore, the results of this report provide an important basis for evaluating the "Sanadak" app and can also provide reference points for other studies with similar 
aims. Overall, participants of the Help@App study showed high global psychological distress across considered outcomes, which highlights the urgent need for intervention.

Author Contributions: Conceptualization: S.R., F.U.J., H.-H.K., A.K., S.G.R.-H.; Methodology, S.R., F.U.J.; Validation, A.R., A.P., R.H., J.D., T.G., H.-H.K., A.K., S.G.R.-H.; Formal Analysis, S.R., F.U.J.; Investigation, S.R., F.U.J., A.R., A.P., R.H., J.D., T.G., H.-H.K., A.K., S.G.R.-H.; Resources, S.G.R.-H.; Data Curation, S.R., F.U.J., T.G.; Writing-Original Draft Preparation, S.R., F.U.J.; Writing-Review \& Editing, A.R., A.P., R.H., J.D., T.G., H.-H.K., A.K., S.G.R.-H.; Visualization, S.R., F.U.J.; Supervision, S.G.R.-H., S.R., H.-H.K., A.K.; Project Administration, S.R., F.U.J., S.G.R.-H.; Funding Acquisition, H.-H.K, A.K., S.G.R.-H. All authors have read and agreed to the published version of the manuscript.

Funding: This research was funded by the "Innovationsfonds" of the German Federal Joint Committee (Gemeinsamer Bundesausschuss/G-BA; grant number: 01VSF16033).

Acknowledgments: This publication is part of the study “HELP@APP: Development and evaluation of a self-help app for traumatized Syrian refugees" and was funded by means of the "Innovationsfonds" of the German Federal Joint Committee (Gemeinsamer Bundesausschuss/G-BA; grant 01VSF16033). The funding source had no involvement in study design, in the collection, analysis and interpretation of data, in the writing of the report, and in the decision to submit the article for publication. The authors would like to thank all study participants for their time and for speaking so openly. Members of the HELP@APP Study Group: Steffi G. Riedel-Heller (Principle Investigator), Anette Kersting (Co-Principal Investigator), Hans-Helmut König (Co-Principal Investigator), Maram Alhamarneh, Mais Alhamwi, Ghadir Ali, Zain Assaad, Ismail Ayoub, Franziska Berg, Franziska Elias, Johannes Golchert, Thomas Grochtdreis, Rahel Hoffmann, David Jäckle, Franziska Jung, Yamen Khamis, Waad Layka, Alia Kheirbek, Chiara Manthey, Amera Mojahed, Michaela Nagl, Anna Plexnies, Anna Renner, Susanne Röhr, Adrian Schultz.

Conflicts of Interest: The authors declare no conflict of interest.

\section{References}

1. Mediendienst Integration. Zahlen Und Fakten: Syrische Flüchtlinge. Available online: https://mediendienstintegration.de/migration/flucht-asyl/syrische-fluechtlinge.html (accessed on 30 April 2020).

2. Georgiadou, E.; Zbidat, A.; Schmitt, G.M.; Erim, Y. Prevalence of Mental Distress among Syrian Refugees With Residence Permission in Germany: A Registry-Based Study. Front. Psychiatry 2018, 9, 393. [CrossRef] [PubMed]

3. Fazel, M.; Wheeler, J.; Danesh, J. Prevalence of serious mental disorder in 7000 refugees resettled in western countries: A systematic review. Lancet 2005, 365, 1309-1314. [CrossRef]

4. PeConga, E.K.; Thøgersen, M.H. Post-traumatic stress disorder, depression, and anxiety in adult Syrian refugees: What do we know? Scand. J. Public Health 2019, 26, 140349481988213. [CrossRef] [PubMed]

5. Lindert, J.; Brähler, E.; Wittig, U.; Mielck, A.; Priebe, S. Depressivität, Angst und posttraumatische Belastungsstörung bei Arbeitsmigranten, Asylbewerbern und Flüchtlingen. PPmP Psychother. Psychosom. Med. Psychol. 2008, 58, 109-122. [CrossRef]

6. Li, S.S.Y.; Liddell, B.J.; Nickerson, A. The Relationship between Post-Migration Stress and Psychological Disorders in Refugees and Asylum Seekers. Curr. Psychiatry Rep. 2016, 18, 1-9. [CrossRef]

7. Association, A.P. (Ed.) Diagnostic and Statistical Manual of Mental Disorders. In Criteria for Post-Traumatic Stress Disorder; American Psychiatric Association: Philadelphia, PA, USA, 2013.

8. Sierau, S.; Schneider, E.; Nesterko, Y.; Von Klitzing, K.; Glaesmer, H. Psychische Belastung bei unbegleiteten jungen Geflüchteten in Jugendhilfeeinrichtungen. Psychiatr. Prax. 2018, 46, 135-140. [CrossRef]

9. Winkler, J.; Brandl, E.J.; Bretz, H.J.; Heinz, A.; Schouler-Ocak, M. Psychische Symptombelastung bei Asylsuchenden in Abhängigkeit vom Aufenthaltsstatus. Psychiatr. Prax. 2018, 46, 191-199. [CrossRef]

10. Wenk-Ansohn, M.; Heeke, C.; Böttche, M.; Stammel, N. Acute short-term multimodal treatment for newly arrived traumatized refugees: Reflections on the practical experience and evaluation. Torture J. 2018, 28, 99-117. [CrossRef]

11. Nesterko, Y.; Jäckle, D.; Friedrich, M.; Holzapfel, L.; Glaesmer, H. Prevalence of post-traumatic stress disorder, depression and somatisation in recently arrived refugees in Germany: An epidemiological study. Epidemiol. Psychiatr. Sci. 2019, 29, e40. [CrossRef]

12. Marshall, G.N.; Schell, T.L.; Elliott, M.N.; Berthold, S.M.; Chun, C.-A. Mental Health of Cambodian Refugees 2 Decades after Resettlement in the United States. JAMA 2005, 294, 571-579. [CrossRef] 
13. Golchert, J.; Röhr, S.; Berg, F.; Grochtdreis, T.; Hoffmann, R.; Jung, F.U.; Nagl, M.; Plexnies, A.; Renner, A.; König, H.-H.; et al. HELP@APP: Development and evaluation of a self-help app for traumatized Syrian refugees in Germany-a study protocol of a randomized controlled trial. BMC Psychiatry 2019, 19, 131. [CrossRef]

14. Röhr, S.; Wittmann, F.; Jung, F.; Hoffmann, R.; Renner, A.; Dams, J.; Grochtdreis, T.; Kersting, A.; König, H.-H.; Riedel-Heller, S.G. Strategien zur Rekrutierung von Geflüchteten für Interventionsstudien: Erkenntnisse aus dem „Sanadak“-Trial. PPmP Psychother. Psychosom. Med. Psychol. 2019, 69, 484-489. [CrossRef] [PubMed]

15. Foa, E.B.; McLean, C.P.; Zang, Y.; Zhong, J.; Powers, M.B.; Kauffman, B.Y.; Rauch, S.; Porter, K.; Knowles, K. Psychometric properties of the Posttraumatic Diagnostic Scale for DSM-5 (PDS-5). Psychol. Assess. 2016, 28, 1166-1171. [CrossRef]

16. Kroenke, K.; Spitzer, R.L.; Williams, J.B.W. The PHQ-9. J. Gen. Intern. Med. 2001, 16, 606-613. [CrossRef]

17. Von Glischinski, M.; Teismann, T.; Prinz, S.; Gebauer, J.E.; Hirschfeld, G. Depressive Symptom Inventory Suicidality Subscale: Optimal Cut Points for Clinical and Non-Clinical Samples. Clin. Psychol. Psychother. 2016, 23, 543-549. [CrossRef]

18. Leppert, K.; Koch, B.; Brähler, E.; Straß, B. Die Resilienzskala (RS)-Überprüfung der Langform RS-25 und einer Kurzform RS-13. Klin. Diagn. Eval. 2008, 1, 226-243.

19. Huber, S.; Huber, O.W. The Centrality of Religiosity Scale (CRS). Religions 2012, 3, 710-724. [CrossRef]

20. Brauns, H.; Steinmann, S. Educational Reform in France, West-Germany and the United Kingdom: Updating the CASMIN Educational Classification. Available online: https://nbn-resolving.org/urn:nbn:de:0168-ssoar208169 (accessed on 30 April 2020).

21. Spitzer, R.L.; Kroenke, K.; Williams, J.B.W.; Löwe, B. A Brief Measure for Assessing Generalized Anxiety Disorder. Arch. Intern. Med. 2006, 166, 1092-1097. [CrossRef]

22. Kroenke, K.; Spitzer, R.L.; Williams, J.B.W. The PHQ-15: Validity of a New Measure for Evaluating the Severity of Somatic Symptoms. Psychosom. Med. 2002, 64, 258-266. [CrossRef] [PubMed]

23. Schwarzer, R.; Jerusalem, M. Skalen zur Erfassung von Lehrer-und Schülermerkmalen. Dokumentation der psychometrischen Verfahren im Rahmen der Wissenschaftlichen Begleitung des Modellversuchs Selbstwirksame Schulen; Freie Universität Berlin: Berlin, Germany, 1999.

24. Corrigan, P.W.; Michaels, P.J.; Vega, E.; Gause, M.; Watson, A.C.; Rüsch, N. Self-stigma of mental illness scale-Short form: Reliability and validity. Psychiatry Res. 2012, 199, 65-69. [CrossRef] [PubMed]

25. Lubben, J.E. Assessing social networks among elderly populations. Fam. Community Health 1988, 11, 42-52. [CrossRef]

26. Kendel, F.; Spaderna, H.; Sieverding, M.; Dunkel, A.; Lehmkuhl, E.; Hetzer, R.; Regitz-Zagrosek, V. Eine deutsche Adaptation des ENRICHD Social Support Inventory (ESSI). Diagnostica 2011, 57, 99-106. [CrossRef]

27. EuroQol Group EuroQol-A new facility for the measurement of health-related quality of life. Health Policy 1990, 16, 199-208. [CrossRef]

28. Kocalevent, R.-D.; Hinz, A.; Braehler, E. Standardization of a screening instrument (PHQ-15) for somatization syndromes in the general population. BMC Psychiatry 2013, 13, 91. [CrossRef] [PubMed]

29. Federal Office for Migration and Refugees. The Stages of the German Asylum Procedure: An Overview of the Individual Procedural Steps and the Legal Basis. Available online: https://www.bamf.de/SharedDocs/Anlagen/EN/AsylFluechtlingsschutz/Asylverfahren/das-deutscheasylverfahren.pdf?_blob=publicationFile\&v=12 (accessed on 30 April 2020).

30. Leiler, A.; Bjärtå, A.; Ekdahl, J.; Wasteson, E. Mental health and quality of life among asylum seekers and refugees living in refugee housing facilities in Sweden. Soc. Psychiatry Psychiatr. Epidemiol. 2018, 54, 543-551. [CrossRef] [PubMed]

31. Euteneuer, F.; Schäfer, S.J. Brief Report: Subjective Social Mobility and Depressive Symptoms in Syrian Refugees to Germany. J. Immigr. Minor. Health 2018, 20, 1533-1536. [CrossRef]

32. Krysinska, K.; Lester, D. Post-Traumatic Stress Disorder and Suicide Risk: A Systematic Review. Arch. Suicide Res. 2010, 14, 1-23. [CrossRef] [PubMed]

33. Amin, M.R.; Helgesson, M.; Runeson, B.; Tinghög, P.; Mehlum, L.; Qin, P.; Holmes, E.A.; Mittendorfer-Rutz, E. Suicide attempt and suicide in refugees in Sweden-A nationwide population-based cohort study. Psychol. Med. 2019, 1-10. [CrossRef] [PubMed] 
34. Björkenstam, E.; Helgesson, M.; Amin, M.R.; Mittendorfer-Rutz, E. Mental disorders, suicide attempt and suicide: Differences in the association in refugees compared with Swedish-born individuals. Br. J. Psychiatry 2019, 352, 1-7. [CrossRef] [PubMed]

35. Henriksson, M.M.; Aro, H.M.; Marttunen, M.J.; E Heikkinen, M.; Isometsä, E.T.; I Kuoppasalmi, K.; Lönnqvist, J.K. Mental disorders and comorbidity in suicide. Am. J. Psychiatry 1993, 150, 935-940. [CrossRef]

36. Scholz, U.; Doña, B.G.; Sud, S.; Schwarzer, R. Is General Self-Efficacy a Universal Construct? Eur. J. Psychol. Assess. 2002, 18, 242-251. [CrossRef]

37. Gottvall, M.; Vaez, M.; Saboonchi, F. Social support attenuates the link between torture exposure and post-traumatic stress disorder among male and female Syrian refugees in Sweden. BMC Int. Health Hum. Rights 2019, 19, 1-11. [CrossRef] [PubMed]

Publisher's Note: MDPI stays neutral with regard to jurisdictional claims in published maps and institutional affiliations.

(C) 2020 by the authors. Licensee MDPI, Basel, Switzerland. This article is an open access article distributed under the terms and conditions of the Creative Commons Attribution (CC BY) license (http://creativecommons.org/licenses/by/4.0/). 